Communicated by A. B. Bonds

\title{
Generation of Direction Selectivity by Isotropic Intracortical Connections
}

\author{
Florentin Wörgötter \\ Institut für Physiologie, Ruhr-Universität Bochum, \\ W-4630 Bochum, Germany \\ Ernst Niebur \\ Christof Koch \\ Computation and Neural Systems Program, California Institute of Technology, \\ Pasadena, CA 91125 USA
}

To what extent do the mechanisms generating different receptive field properties of neurons depend on each other? We investigated this question theoretically within the context of orientation and direction tuning of simple cells in the mammalian visual cortex. In our model a cortical cell of the "simple" type receives its orientation tuning by afferent convergence of aligned receptive fields of the lateral geniculate nucleus (Hubel and Wiesel 1962). We sharpen this orientation bias by postulating a special type of radially symmetric long-range lateral inhibition called circular inhibition. Surprisingly, this isotropic mechanism leads to the emergence of a strong bias for the direction of motion of a bar. We show that this directional anisotropy is neither caused by the probabilistic nature of the connections nor is it a consequence of the specific columnar structure chosen but that it is an inherent feature of the architecture of visual cortex.

All of the response properties of cortical cells can in principle be explained with models that postulate a high degree of connection specificity. Establishing highly specific connections requires, however, large amounts of information, which might be more than what can be determined genetically or learned during development. Thus, it becomes of interest to investigate the extent to which unspecific mechanisms, which can be established with minimal information requirements, underlie the observed receptive field properties.

We constructed over the last years a detailed model of the connectivity in a small patch in layer IV of cat visual cortex in order to investigate the mechanisms underlying orientation selectivity. The model of a $5^{\circ} \times 5^{\circ}$ patch of the primary visual pathway of cat includes a total of more than 16,000 cells in the ON and OFF subsystems of retina and LGN and simple

Neural Computation 4, 332-340 (1992) (C) 1992 Massachusetts Institute of Technology 
cells in layer IV in area 17 . The retina is stimulated with moving light bars. Cells are modeled as improved integrate-and-fire neurons. Our single-cell model takes into account absolute and relative refractory periods and the after-hyperpolarization following a spike, but it stops short of the fine details that can be found, for instance, in the Hodgkin-Huxley model. Realistic convergence and divergence numbers between cell populations are implemented using more than 2,000,000 synapses (Orban 1984). Each cortical cell receives input from a field of (on average) $5 \times 13$ LGN cells. This convergence of LGN receptive fields leads to an initial orientation bias with a cortical receptive field elongation of 1.7 . The preferred orientation of the cortical receptive fields is changed continuously across the modeled patch with a periodicity (hypercolumn width $\lambda$ ) of $1^{\circ}$. Receptive fields are modeled with realistic scatter and jitter (Albus 1975) and axonal delays were set to realistic values (Hoffmann et al. 1972). All parameters are subject to statistical fluctuations (Wehmeier et al. 1989; Wörgötter and Koch 1991). Figure 1B shows the average orientation and direction tuning curve of 55 randomly chosen cells from this model obtained by using only a Hubel and Wiesel type wiring scheme (Hubel and Wiesel 1962; see Chapman et al. for some recent evidence supporting this model). Orientation tuning is conferred on these cells by virtue of this specific type of afferent wiring from LGN to cortex. Another possibility to achieve an orientation bias would be to use elongated LGN receptive fields (Vidyasagar 1984; Vidyasagar and Urbas 1982; Shou and Leventhal 1989; Soodak et al. 1987). The actual mechanism underlying the orientation bias does not, however, influence the central result of this study and, therefore, we chose to use the rather well-known Hubel and Wiesel connection scheme. Figure $1 \mathrm{C}$ shows the orientation tuning after "circular inhibition" (Niebur and Wörgötter 1990) is superimposed onto the afferent bias. In this scheme, cortical interneurons preferentially inhibit cells located a certain distance away (Fig. 1A). Wörgötter et al. (1991) showed analytically that at a radius of half a hypercolumn $(\lambda / 2)$, circular inhibition acts as a weakly tuned cross-orientation inhibition (Benevento et al. 1972) for most cells, because $(\lambda / 2)$ is the smallest radius at which cells with orthogonal orientation preferences contribute inhibition. This effect, however, is not strong, because inhibition from many orientations is included. Circular inhibition not only results in an increase in orientation tuning, but also in a marked increase in direction tuning, which attains ${ }^{1}$ $D=23 \%(D I=44 \%)$. Some degree of direction tuning is expected for any connection scheme with probabilistic variability, since the net synaptic input to a cell from a given direction will, in general, not be identical to that from the opposite direction. In our simulation, "random" inhibition

\footnotetext{
${ }^{1}$ The direction index $D I$ is based on the difference of cell responses only along the axis of preferred direction (Orban 1984). A more reliable measure for direction selectivity that takes into account the cell response for all directions is $D$, the first moment of the statistical distribution of responses (Swindale et al. 1987; Wörgötter et al. 1990). We will always give values of $D$ and $D I$ in the text.
} 


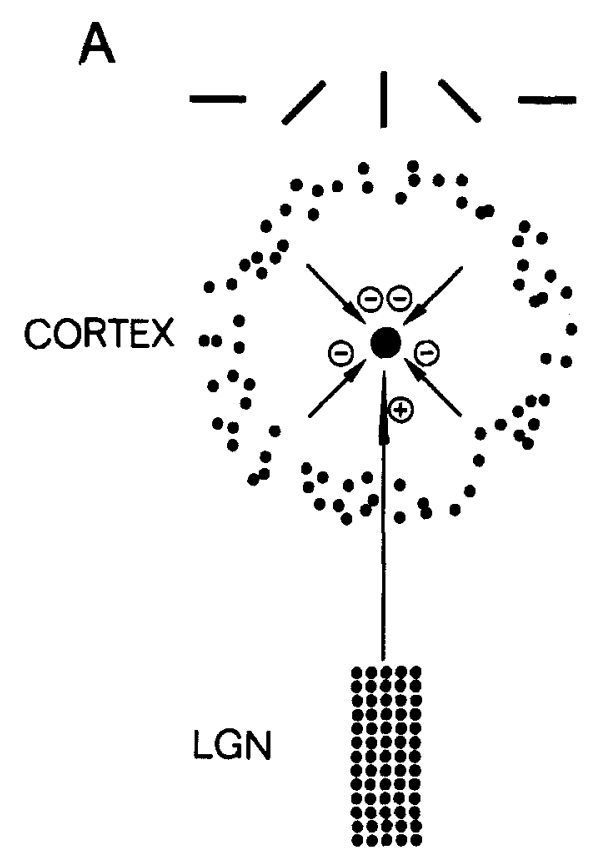

B
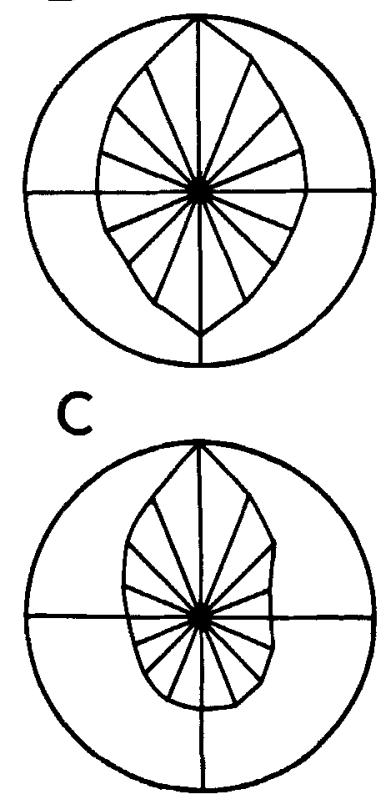

Figure 1: Direction tuning arising from an isotropic connection scheme. (A) Part of the connection diagram for cortical cells in the detailed cortex model (Wehmeier et al. 1989; Wörgötter and Koch 1991). Part of the column structure is depicted on top. Orientation columns run parallel to the $y$-axis in the simulated patch. Each cortical cell receives inhibitory input from other cortical cells located at a distance of about half a hypercolumn ("circular inhibition"). On average 100 cortical cells converge onto each target cell. (B) Average direction tuning curve obtained with LGN convergence but without intracortical inhibition. Peak impulse rates are plotted as a function of the stimulus angle $\alpha$ after rotation of all polar plots to a common preferred orientation. Without intracortical inhibition, $D=8 \%(D I=16 \%)$. (C) Average tuning curve after including circular inhibition. Although circular inhibition consists of isotropic connections it leads to a clear direction bias $(D=23 \%, D I=44 \%)$.

arising from about 200 cells randomly selected within a certain distance from the target cell leads to a direction tuning of $D=12 \%(D I=28 \%)$ (see Wörgötter and Koch 1991). These probabilistic effects are, however, small compared to the strong increase in $D$ observed with circular inhibition. How does this directional anisotropy arise from isotropic intracortical connections? Obviously, the cortical column structure, which is 
predetermined by the afferent orientation bias, must supply the necessary anisotropy.

A better understanding of this effect is desirable than that which is obtained from the detailed simulation in which the phenomenon is obscured by the large number of parameters. Therefore, we developed a different model, stripping the cells from all properties except their orientation tuning. A cell is then completely described by equation 1.1 below. In Figure 2A, a part of the simplified cortical column structure is shown. Short lines indicate the preferred orientation $\phi$ of cortical cells, described by $\phi(x)=\pi(x / \lambda)$. In this equation, $x$ is the spatial coordinate along the horizontal axis. The target cell in the center receives inhibitory input from all cells located on a circle with a radius $r$ of half a hypercolumn, $r=\lambda / 2$. A cell with preferred orientation $\phi$ is assumed to respond with the activity function

$$
C(\gamma-\phi)=C_{0}+C_{2} \cos (2 \gamma-2 \phi)
$$

to a stimulus bar with angle $\gamma$ across the receptive field of the cell, where $C_{0}$ and $C_{2}$ are constants satisfying $C_{0} \geq C_{2}>0$. Equation 1.1 is a simple model for an orientation selective cell with preferred orientation $\phi$. The response of this cell is maximal $\left(C_{0}+C_{2}\right)$, if the stimulus is aligned with the preferred orientation (i.e., $\gamma=\phi)$ and minimal $\left(C_{0}-C_{2}\right)$, if the orientation of the stimulus is orthogonal to the preferred orientation of the cell (i.e., $\gamma=\phi \pm \pi / 2$ ). The half-width-at-half-height of the orientation tuning is adjusted to match the average orientation tuning of cortical simple cells (Orban 1984). A retinotopic projection is assumed to exist over distances of $>200 \mu \mathrm{m}$, as has been observed experimentally (Albus 1975), but not necessarily over much shorter distances. We restrict ourselves to the treatment of inhibitory intracortical connections, because the generation of an anisotropic directional effect from isotropic connections is not dependent on the sign of the interaction. We assume that the inhibitory input to the target cell arises from the cell on the circle that is excited first by the moving stimulus bar. We will further assume that, due to a fixed axonal propagation delay, this inhibition arrives within a small time window together with the activation of the center cell. Thereby we neglect effects induced by different stimulus velocities. None of the above assumptions influences the qualitative observation of the generation of a direction bias as such but only its actual strength (e.g., see Fig. 2D for the radius dependency). The inhibition elicited by a certain stimulus is given by the activity function defined above. A graphic representation of this inhibition is provided by the length of the cross section that cuts through the polar-plot along the stimulus orientation. The black bars inside the polar-plots in Figure 2B show the amount of inhibition for eight example stimuli with different orientations. Plotting the strength of inhibition against the direction of the stimulus motion reveals the tuning of inhibition of a particular cell (Fig. 2C). The diagram shows that motion with a downward component elicits significantly less inhibition than motion 
A

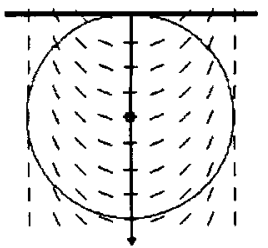

C

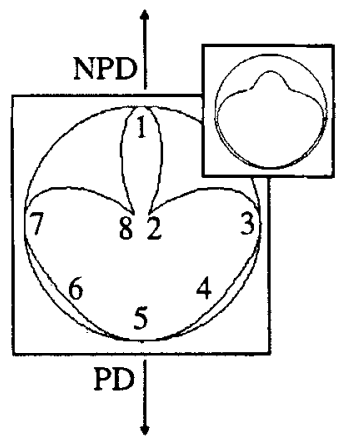

B

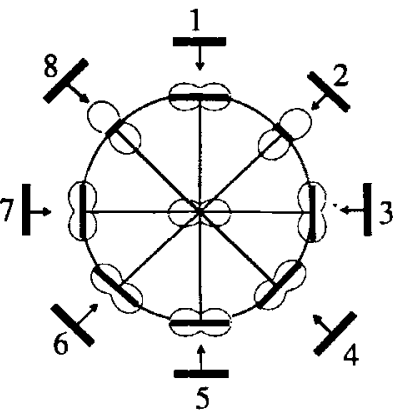

D

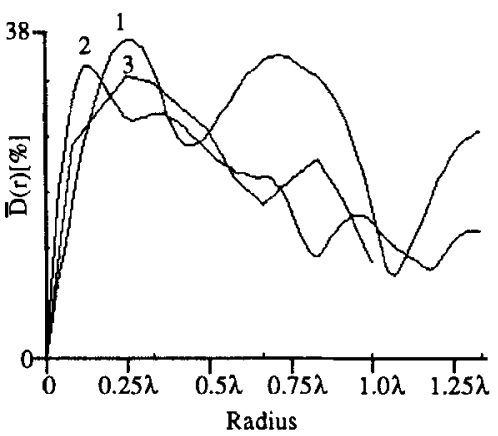

Figure 2: Structural model for the explanation of the direction bias generated by circular inhibition. (A) Part of the simplified cortical column structure. Short lines indicate the preferred orientation $\phi$ of cortical cells. The target cell in the center receives inhibitory input from all cells located on a circle with a radius of half a hypercolumn $(r=\lambda / 2)$. A cell with preferred orientation $\phi$ is assumed to respond with the activity function $C(\gamma-\phi)=C_{0}+C_{2} \cos (2 \gamma-2 \phi)$ to a stimulus bar with angle $\gamma$ across the receptive field of the cell (elliptical tuning curves with "wasp waist," half-width-at-half-height orientation tuning $\approx 20^{\circ}$ ). (B) Stimuli with eight different orientations elicit inhibition defined as the cross section of the moving bar through the receptive field center (black bars inside the tuning curves). (C) Tuning of inhibition for a cell with horizontal preferred orientation obtained by plotting the lengths of the cross sections against the angle of stimulus motion. Inset: Tuning of inhibition for the same cell including jitter in the orientation columns and using a $0.5^{\circ}$ wide bar. (D) Radius dependency of the population average direction bias $\bar{D}$. We compute $\bar{D}$ as average over the $D$ values for cells with all preferred orientations. Curves are shown for straight (curve 1) and realistically bended (curve 2) columns as well as for a patch of an observed cortical column structure [see ref. (Swindale et al. 1987), curve 3]. The equation for the generation of bended columns is given elsewhere (Niebur et al. 1991; Wörgötter et al. 1991). The curves are qualitatively similar for $r<\lambda / 2$ and give values for $\bar{D}(r)$ of up to $38 \%(\overline{D I}=57 \%)$. 
with an upward component, resulting in an average $D$ value of $36 \%$. It is worth emphasizing that the anisotropy of the tuning curve (Fig. $2 \mathrm{C}$ ) is not due to probabilistic effects, since this model is noise free. The amount of inhibition arising exactly along the axis of preferred motion is identical for both directions. This singular situation occurs only in the oversimplified straight column structure. Including realistic jitter of $\pm 10^{\circ}$ into the columns (Albus 1975) and using a $0.5^{\circ}$ wide bar instead of an infinitely narrow bar increases the direction tuning and removes the singular point (see inset in Fig. 2C).

The direction bias of individual cells depends on their location in the column structure and can disappear completely at certain locations. Therefore, the average direction bias of the whole cell population, $\bar{D}$, gives a better estimate of the overall effect. In Figure 2D the radius for circular inhibition is varied and the radius dependency of $\bar{D}$ is shown for more realistic columnar structures. Curve 1 was obtained from the straight column structure discussed so far; curve 2 from a realistically curved column structure (Niebur et al. 1991; Wörgötter et al. 1991), and curve 3 belongs to an observed cortical patch in area 18 of the cat, described by Swindale et al. (1987). Up to a radius of half a hypercolumn $(r \approx \lambda / 2)$ all curves yield a similar average direction bias. This justifies the analysis of the simple straight column structure. Note that the average direction bias $(\bar{D}=26 \%$ or $\overline{D I}=47 \%)$ is close to the average direction bias observed in simple cells $[\bar{D} \approx 28 \%$ or $\overline{D I} \approx 50 \%$ (Orban 1984; Berman et al. 1987)]. Although exact quantitative statements are not possible with our simple model, the strength of this effect cannot be neglected.

We introduced circular inhibition as an unspecific connection scheme allowing us to investigate the limits of specificity necessary to achieve functional order. Circular inhibition sharpens orientation tuning (Wörgötter and Koch 1991; Wörgötter et al. 1991); but, similar to cross-orientation inhibition, circular inhibition cannot generate orientation tuning without an initial orientation bias. Direction tuning, however, arises without any preexisting direction bias.

It seems plausible that such a readily available direction bias is used in development and strengthened by enhancing those mechanisms that add to its performance. Such an interpretation is supported by the experimental finding that during development, only a small number of cells are initially direction selective. Many more cells are only weakly biased early in development and their direction selectivity increases only after the development of orientation tuning (Grigonis et al. 1988). This would support our notion that direction tuning follows the emergence of orientation selectivity.

Our results do not contradict findings that direction tuning can be elicited over short distances (Ganz and Felder 1984). One should, however, note that such short-range connections have to be specifically designed for the generation of direction tuning, whereas we have shown that even unspecific long-range connections will result in a direction bias. 
Very little connection information (radius and annulus diameter) is necessary to establish circular inhibition, which can be considered as a particular type of long-range lateral inhibition. Thus, circular inhibition represents a rather unspecific and broadly tuned connection scheme that appears easy to implement developmentally. In fact, weak tuning of inhibition compatible with this scheme has been observed recently (Bonds 1989). However, the rigidly defined circular inhibition used in this study is a rather unrealistic connection scheme. Does the generation of a directional anisotropy depend on the details of this scheme? In the following, we will show that this is not the case and that our result is valid for a much larger class of systems than the column structures and connection schemes we have considered thus far. We will show that directional anisotropy can be expected to occur in all realistic column structures and all realistic long-range connection schemes.

One key element necessary for the emergence of anisotropy is the inhomogeneity of the orientation column structure, by which the preferred orientations change systcmatically along the cortex. As a result, any two cells with a sufficiently large distance between them are likely to have different orientation tuning. Consequently, connections originating from those cells (i.e., long-range connections ${ }^{2}$ ) will have different impact on the target cell and, thus, produce directional tuning. Without further assumptions on the distribution of the connections, this effect will be reasonably strong only if the number of converging cells is small. The reason is that even if the number of connections is large and they are spread out at random, the contributions will average out to a large extent and only a small anisotropy will be the result. This is avoided if the connections are not distributed at random, but "clustered" in sufficiently small areas (Gilbert and Wiesel 1983), each one encompassing less than the full range of orientations.

In summary, locally correlated activity with a drop-off of the correlation at larger distances and clustered connections with a sufficiently large distance between the clusters is all that is needed to generate direction tuning from any realistic (i.e., containing large cell numbers) long-range connection scheme. Note that the term "cluster" should by no means be interpreted in a narrow sense, for example, in the case of circular inhibition, there is basically only one "cluster," namely the circle of connections around the target cell. This shows that circular inhibition is, in a sense, a "worst-case scenario," because it is an isotropic connection pattern. Furthermore, the direction tuning arising from the interaction of clustered synaptic connections and an underlying inhomogeneous column structure is not limited to orientation selective cells. This could provide an explanation for the direction selectivity that is observed in response to nonoriented stimuli, like random dots (Hammond 1978).

\footnotetext{
${ }^{2}$ Obviously, "long-range connection" refers to a connection whose length is at least comparable to the distances over which the preferred orientation changes appreciably, i.e., a hypercolumn. This may be taken as the definition of the term "long-range."
} 
Thus, it seems that the columnar organization of the cortex, together with basically all long-range connection schemes (in the sense defined above), leads inevitably to the emergence of direction selectivity. The search for specific intracortical mechanisms for direction tuning might, therefore, be in vain.

\section{Acknowledgments}

We thank Dr. J. Knierim for a critical reading of the manuscript. F. W. is supported by the Deutsche Forschungsgemeinschaft, E. N. by the Swiss National Science Foundation, Grant 8220-2594. C. K. acknowledges the support of the Air Force Office of Scientific Research, a NSF Presidential Young Investigator Award, and the James S. McDonnell Foundation.

\section{References}

Albus, K. 1975. Exp. Brain Res. 24, 181-202.

Benevento, L. A., Creutzfeldt, O. D., and Kuhnt, U. 1972. Nature (London) 238, 124-126.

Berman, N. E. J., Wilkes, M. E., and Payne, B. R. 1987. J. Neurophysiol. 38, 676-699.

Bonds, A. B. 1989. Vis. Neurosci. 2, 41-55.

Chapman, B., Zahs, K. R., and Stryker, M. P. 1991. J. Neurosci. 11(5), 1347-1358.

Ganz, L., and Felder, R. 1984. J. Neurophysiol. 51, 294-324.

Gilbert, C. D., and Wiesel, T. N. 1983. J. Neurosci. 3, 1116-1133.

Grigonis, A. M., Zingaro, G. J., and Murphy, E. H. 1988. Dev. Brain Res. 40, 315-318.

Hammond, P. 1978. J. Physiol. (London) 285, 479-491.

Hoffmann, K. P., Stone, J., and Sherman, S. M. 1972. J. Neurophysiol. 35, 518-531. Hubel, D. H., and Wiesel, T. N. 1962. J. Physiol. (London) 160, 106-154.

Niebur, E., and Wörgötter, F. In Proceedings of the IJCNN'90, San Diego, pp. II367-II-372. IEEE Press, Piscataway, NJ.

Niebur, E., Wörgötter, F., and Koch, C. 1991. In Proceedings of the 3rd Midwestern Conference on Neural Networks, S. Sayegh, ed., Purdue Research Foundation Press, W. Lafayette, pp. 67-74.

Orban, G. A. 1984. Neuronal Operations in the Visual Cortex. Springer, Berlin.

Shou, T., and Leventhal, A. G. 1989. J. Neurosci. 9, 4287-4302.

Soodak, R. E., Shapley, R. M., and Kaplan, E. 1987. J. Neurophysiol. 58, 267-275.

Swindale, N. V., Matsubara, J. A., and Cynader, M. S. 1987. J. Neurosci 7, 1414 1427.

Vidyasagar, T. R. 1984. Exp. Brain Res. 55, 192-195.

Vidyasagar, T. R., and Urbas, J. V. 1982. Exp. Brain Res. 46, 157-169. 
Wehmeier, U., Dong, D., Koch, C., and Van Essen, D. 1989. In Methods in Neuronal Modeling, C. Koch and I. Segev, eds., pp. 335-359. MIT Press, Cambridge.

Wörgötter, F., Gründel, O., and Eysel, U. T. 1990. Eur. J. Neurosci. 2, 928-941.

Wörgötter, F., and Koch, C. 1991. J. Neurosci., 11(7), 1959-1979.

Wörgötter, F., Niebur, E., and Koch, C. 1991. J. Neurophysiol. 66(2), 444-459.

Received 3 May 1991; accepted 7 November 1991. 